(c) American Dairy Science Association, 2004.

\title{
Inbreeding Trends and Application of Optimized Selection in the UK Holstein Population
}

\author{
J. F. Kearney, E. Wall, B. Villanueva, and M. P. Coffey \\ Scottish Agricultural College, \\ Edinburgh, EH9 3JG, Scotland, UK
}

\begin{abstract}
Important increases in the rates of inbreeding have recently been observed in dairy cattle populations, and methods have been proposed to address these increases. The aims of this study were to estimate the current level and rates of inbreeding in the UK Holstein population and to investigate the potential of applying optimized selection to manage the rates of inbreeding. Inbreeding coefficients were calculated for the entire UK Holstein population using 1940 as the base year. Rates of inbreeding were obtained for 3 time periods by regressing mean inbreeding coefficients on the year of birth of the animals. The expected average pedigree index and expected inbreeding of offspring using optimized contributions for a given set of selection candidates was compared to the expected pedigree index and inbreeding of offspring for the same set of selection candidates using observed contributions. The rate of inbreeding in the UK Holstein population has increased substantially since 1990 when compared to previous time periods. This increase is most likely due to the large influence of a few related sires on the breed in the mid- to late 1980s. The introduction of the individual animal model in the early 1990s may also have contributed to increased inbreeding. Optimized selection appears to represent a promising selection tool, not only to manage rates of inbreeding, but also to increase genetic gain at the same rate of inbreeding.
\end{abstract}

(Key words: inbreeding, Holstein, optimized selection)

Abbreviation key: £PLI = profitable life index, $£ \mathbf{P I N}=$ production profit index, $\Delta \mathbf{F}=$ difference between offspring and parent average inbreeding coefficients, Top $\mathbf{2 0 0 0}=$ parents of the top 2000 males born in 2002, Top $1000=$ parents of the top 1000 males born in 2002 .

Received March 8, 2004.

Accepted May 5, 2004.

Corresponding author: J. F. Kearney; e-mail: f.kearney@ed.sac. ac.uk.

\section{INTRODUCTION}

Despite being a numerically large breed, inbreeding of Holstein populations is increasing and becoming a concern in many countries. In the United States, the current inbreeding coefficient of Holstein cows is 5\% (AIPL, 2003), representing a doubling since 1990. In Canada, the current average inbreeding is $4.91 \%$, and the rate of increase has been $0.25 \% / y r$ from 1990 to 2000, a fivefold increase when compared to the previous decade (CDN, 2003). Estimates of average inbreeding and rates of inbreeding for the UK dairy population are not routinely published. Roughsedge et al. (1999) reported an average inbreeding coefficient of $0.43 \%$ for the British Holstein-Friesian population for animals born in 1997. However, these results were based on multiple random samples of 2000 cows rather than the whole pedigree and, more importantly, there was incomplete pedigree information for foreign sires.

The increase in inbreeding in the Holstein populations can be attributed to a number of factors including 1) the tendency to coselect related animals as a result of using BLUP estimated breeding values, 2) the use of fewer sires and dams facilitated by AI and multiple ovulation and embryo transfer, and 3) selection for only a few traits such as milk yield and type, which are usually positively correlated. For example, Young and Seykora (1996) identified 2 sires that together accounted for nearly one-quarter of the genes of registered US Holstein animals born in 1990. They are still the 2 most highly related sires to Holstein cows in the United States (AIPL, 2003), and were recently reported as appearing in more than $95 \%$ of all pedigrees of inbred German Holstein cows (Swalve et al., 2003).

The consequences of inbreeding are manifested in terms of inbreeding depression, an increase in undesirable recessive disorders, and a loss in genetic variation. Numerous studies have shown an unfavorable association between performance for production traits (e.g., Smith et al., 1998; Thompson et al., 2000) and nonproduction traits (e.g., Wall et al. 2003; Cassell et al., 2003) with increasing inbreeding. Similarly, the prevalence of known genetic disorders, such as complex vertebral malformation (CVM) and bovine leucocyte adhesion de- 
ficiency (BLAD), and the appearance of other new disorders may increase as inbreeding becomes more widespread. These consequences have the potential to be expensive in terms of production losses due to inbreeding depression and veterinary and culling costs associated with genetic disorders. Also, the loss of genetic variation as a result of inbreeding is important, as it limits the ability to improve traits through genetic selection.

Several strategies to control the rate at which inbreeding accumulates have been proposed, including the modification of mating schemes (e.g., avoiding mating of relatives), modifications to the BLUP procedure, such as artificially inflating the estimate of heritability and the use of optimized contributions (see Weigel, 2001; Villanueva et al., 2004 for reviews). The potential application of optimized contributions in real livestock populations has been investigated. Weigel and Lin (2002) concluded that optimized selection could be used to control the rate of inbreeding in the US dairy cattle populations, but did not compare the genetic gain achieved by optimized selection to current genetic gain. In a study of a beef and sheep population, Avendaño et al. (2003) found that optimized selection can lead to increased genetic gains of 17 (beef) and 30\% (sheep) when compared to conventional BLUP truncation selection at the same rate of inbreeding.

The objective of this study was to determine the current level and rates of inbreeding and to assess the potential of using optimized selection procedures in the UK dairy population. It is expected that the bull breeding path of selection will control the long-term genetic response and rates of inbreeding in the whole population (Goddard and Smith, 1990; Weigel and Lin, 2002); therefore, we concentrate on using optimized selection to manage the contributions of parents of young bulls that would be entering progeny testing schemes.

\section{MATERIALS AND METHODS}

\section{Inbreeding}

Pedigrees were extracted from the Holstein UK database for animals born since 1940. The database includes all registered pedigree and grading up animals and all imported foreign males and females with progeny in the United Kingdom. Percentage Holstein was also available for each animal. Inbreeding coefficients for each animal were calculated using the algorithm of Meuwissen and Luo (1992) for 330,037 males and $7,029,545$ females born from 1940 to 2002 . Mean inbreeding was calculated per year based on the year of birth of the animals. To assess the quality of the data to estimate inbreeding, a measure of pedigree completeness was calculated for each animal according to Mac-
Cluer et al. (1983). In 2002, 96\% of animals had 3 or more generations of complete pedigree information, and $85 \%$ had 4 or more generations of complete information. The rate of inbreeding was calculated by regressing the mean inbreeding on the year of birth for 3 time periods: 1) 1940 to 1968, 2) 1968 to 1991, and 3) 1992 to 2002.

\section{Optimized Selection}

The approach of Meuwissen (1997) with the constraint on the rate of inbreeding of Grundy et al. (1998) was used to calculate the number of males and females and their respective contributions (c) that maximize genetic gain, while constraining the rate of inbreeding to a predefined level. A vector ( $\mathbf{g}$ ) of PTA from the August 2003 genetic evaluation for the selection candidates was constructed for the 2 indices currently used in the UK: production profit index (£PIN) and profitable life index (£PLI) (MDC, 2003). The £PLI includes indicators of longevity in addition to production. The 2 indices were analyzed to determine how important the breeding objective is when using optimized selection. The algorithm maximizes genetic gain, c'g, subject to 2 restrictions. The first is a restriction on the rate of inbreeding, whereas the second restriction ensures that the sum of male and female selection candidate contributions equal one half each.

Two types of optimizations were undertaken. The first optimization was when no constraint was placed on the contribution of selection candidates. In this situation, it is assumed that there is no restriction on the reproductive capacity of either sex, such that males can contribute unlimited semen and cows can contribute unlimited ova. In the second optimization, all females were selected, and only male contributions were optimized.

Two groups of selection candidates were considered. In the first group, the selection candidates were the parents of the top 2000 males (Top 2000) born in 2002, and in the second group, the selection candidates were parents of the top 1000 males (Top 1000) born in 2002.

Males born in 2002 were ranked on their pedigree index value for £PLI or £PIN. The pedigree index values were based on the August 2003 genetic evaluation of their parents. These males represent the result of actual selection decisions made in the previous generation and are the bulls that are available to breeding companies for potential progeny testing. Both male and female selection candidates were required to have at least 4 generations of complete pedigree recorded.

The benefits of using optimized selection were assessed by comparing the expected average pedigree index using optimized contributions to the expected average pedigree index using observed contributions at the 


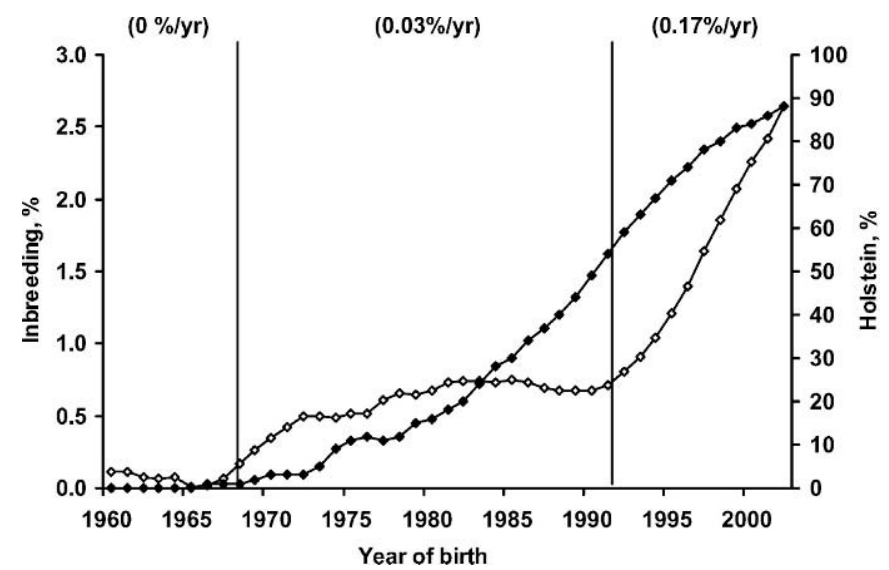

Figure 1. Average inbreeding coefficients $(\diamond)$, percentage Holstein $(\diamond)$, and rates of inbreeding (in parentheses) for the UK dairy population.

same level of inbreeding. To do this, the observed contributions of parents were calculated based on the number of offspring born. These observed contributions were used to determine the expected pedigree index and level of inbreeding of their offspring. Optimized selection was then used for the same set of parents to calculate optimum contributions and the expected average pedigree index of offspring. Optimized selection for the 2 indices (£PLI and £PIN) and 2 groups of selection candidates (Top 2000 and Top 1000) was investigated for 5 levels of constraint on inbreeding.

\section{RESULTS}

The base population for the analysis of inbreeding was 1940 . The rate of inbreeding in the population was effectively zero until 1960, so the results reported here describe the population trends since 1960. Mean inbreeding coefficients and rates of inbreeding are shown in Figure 1. The increase in inbreeding was nonlinear in the whole period evaluated, and 3 distinct time periods were considered in terms of the rate of inbreeding. From 1940 to 1967 there was no increase in the rate of inbreeding. There was a slight increase $(0.03 \% / y r)$ from 1968 to 1991, but since 1992, inbreeding has increased at a rate of $0.17 \% / y r$. In 2002 , the average inbreeding was $2.64 \%$ for females and $3.06 \%$ for males. The number of animals inbred and the number in higher inbreeding classes have increased significantly since 1990 (Table 1). Currently, $98 \%$ of all males and $96 \%$ of all females are inbred to some degree, compared with approximately 50\% in 1990 (Table 1). The increase in inbreeding has coincided with an increase in the percentage of Holstein in the population (Figure 1). A slight decrease in inbreeding occurred around the late 1980s and early
Table 1. Frequency of males and females by level of inbreeding (F) for animals born in 1990 and 2002 .

\begin{tabular}{llllll}
\hline & \multicolumn{2}{c}{ Males } & & \multicolumn{2}{c}{ Females } \\
\cline { 2 - 3 } \cline { 5 - 6 } Inbreeding \% & 1990 & 2002 & & 1990 & 2002 \\
\hline $\mathrm{F}=0$ & 0.474 & 0.015 & & 0.497 & 0.040 \\
$0<\mathrm{F} \leq 6.25$ & 0.502 & 0.910 & & 0.486 & 0.907 \\
$6.25<\mathrm{F} \leq 12.5$ & 0.021 & 0.066 & & 0.012 & 0.045 \\
$12.5<\mathrm{F} \leq 25$ & 0.003 & 0.009 & & 0.005 & 0.006 \\
$\mathrm{~F}>25$ & 0 & 0 & & 0 & 0.002 \\
\hline
\end{tabular}

1990s as the population of then mainly British Friesian cows were mated to imported Holstein sires. However, influential Holstein sires quickly became established in the United Kingdom and were used extensively in the early 1990s. During this time, the average relationship of the top 10 most widely used paternal grandsires rose from $2 \%$ in 1987 to almost $12 \%$ in 1992 (results not shown), with some sires having 40,000 granddaughters or more entering the recorded national herd each year.

A summary of the number, mean £PLI and £PIN of male and female selection candidates appears in Table 2 . The average relationship between the males and females candidates was 6.6 and $7.3 \%$ for Top 2000 and Top 1000, respectively. For clarity, the following results relate to the analysis of Top 2000 for £PLI. Results for the other data sets are similar and are therefore not presented. Expected average pedigree index and inbreeding using the observed mating contributions and optimum contributions are shown in Table 3. For the purpose of these results, the difference between the average inbreeding coefficient of the offspring, compared with the average inbreeding coefficient of the parents will be noted by $\Delta \mathbf{F}$. The expected $\Delta \mathrm{F}$ resulting from the observed contributions was $2.0 \%$, and the expected £PLI of the offspring was $£ 62$. Optimizing contributions of the selection candidates to attain the observed $\Delta \mathrm{F}$ (i.e., $\Delta \mathrm{F}=2 \%$ ) resulted in an average $£ \mathrm{PLI}$ of $£ 72$ when male contributions only were optimized and $£ 102$ when the contributions of both sexes were optimized. This represents an increase in genetic gain of 16 and $62 \%$, respectively, over current gain when using optimized selection.

Table 2. Summary data for the 2 groups of selection candidates.

\begin{tabular}{|c|c|c|c|c|c|c|}
\hline & \multicolumn{3}{|c|}{ Top 2000} & \multicolumn{3}{|c|}{ Top 1000} \\
\hline & No. ${ }^{1}$ & $£ \mathrm{PLI}^{2}$ & $£ \mathrm{PIN}^{3}$ & No. & $£$ PLI & $£ P I N$ \\
\hline Males & 173 & 56 & 54 & 76 & 64 & 63 \\
\hline Females & 1642 & 52 & 51 & 729 & 65 & 65 \\
\hline
\end{tabular}

${ }^{1}$ No. $=$ Number of male and female parents.

${ }^{2} £$ PLI $=$ Average $£$ PLI index scores of parents.

${ }^{3} £$ PIN $=$ Average $£$ PIN index scores of parents. 
Table 3. Rate of inbreeding $(\Delta \mathrm{F})$, expected average index score, and number of selected males and females from observed and optimized contributions for £PLI for Top 2000.

\begin{tabular}{|c|c|c|c|c|c|c|c|c|c|c|c|}
\hline \multirow[b]{3}{*}{$\Delta \mathrm{F}(\%)^{2}$} & \multirow{3}{*}{$\frac{\text { Observed }}{2}$} & \multicolumn{10}{|c|}{ Optimized selection ${ }^{1}$} \\
\hline & & \multicolumn{5}{|c|}{ Males only } & \multicolumn{5}{|c|}{ Both sexes } \\
\hline & & 0.1 & 0.2 & 0.5 & 1 & 2 & 0.1 & 0.2 & 0.5 & 1 & 2 \\
\hline$£ \mathrm{PLI}^{3}$ & 62 & 60 & 62 & 65 & 68 & 72 & 90 & 91 & 93 & 97 & 102 \\
\hline No. males ${ }^{4}$ & 173 & 46 & 44 & 39 & 31 & 11 & 42 & 41 & 39 & 34 & 25 \\
\hline No. females ${ }^{5}$ & 1642 & 1642 & 1642 & 1642 & 1642 & 1642 & 47 & 46 & 39 & 34 & 24 \\
\hline
\end{tabular}

Optimized selection was successful in constraining $\Delta \mathrm{F}$ to the predefined levels. As expected, the number of animals required to have nonzero contributions increased when more severe constraints were placed on $\Delta \mathrm{F}$. For example, when contributions of both sexes were optimized, the total number of animals required increased from 49 ( 25 males and 24 females) to 89 (42 males and 47 females) as $\Delta \mathrm{F}$ was reduced from 2 to $0.1 \%$. Similar numbers of males and females were required to meet the constraint at each level. When only male contributions were optimized, 11 sires were required at $\Delta \mathrm{F}=2 \%$, increasing to 46 for $\Delta \mathrm{F}=0.1 \%$. Forty-four sires were required to achieve the same genetic gain as the observed 173 sires, but $\Delta \mathrm{F}$ in the offspring was reduced 10 -fold $(\Delta \mathrm{F}=0.2 \%)$. At each level of $\Delta \mathrm{F}$, more animals were required to achieve an optimal solution when the index under consideration was £PLI compared with £PIN. Optimization on £PIN led to $7,6,3$, and 2 sires fewer than $£$ PLI for $\Delta \mathrm{F}=0.1$, $0.2,0.5$, and $1 \%$, respectively, when only contributions of males were optimized (results not shown). Equal numbers were selected at $\Delta \mathrm{F}=2 \%$. This is most likely due to reranking of the sires for the 2 indices.

Figure 2 shows the relationship between optimal contributions and index scores (£PLI) of selected males for
3 levels of $\Delta \mathrm{F}$. As the constraint is relaxed, fewer sires are required, differences in contributions among selected sires increase, and there is a much stronger association between the contributions and £PLI. At the most relaxed constraint $(\Delta \mathrm{F}=2 \%)$, the individuals with the highest £PLI have clearly the highest contributions. However, when $\Delta \mathrm{F}$ is constrained to $0.2 \%$, the males with the highest £PLI do not necessarily have the highest contributions.

\section{DISCUSSION}

The rate of inbreeding in the UK Holstein population has increased considerably in the last decade. Roughsedge et al. (1999) estimated a mean inbreeding coefficient of $0.43 \%$ for animals born in 1997, with a base year of 1960. For the same year, our estimated mean inbreeding coefficient was considerably higher $(1.64 \%)$. More interestingly, the rate of increase in inbreeding from 1992 to 1997 was only about $0.01 \% / y r$ (Roughsedge et al., 1999) compared with $0.17 \% / y r$ in this study. These differences may be attributable to the different base years, the use of random samples, and missing pedigree information (in particular from foreign sires) in the study of Roughsedge et al. (1999). The
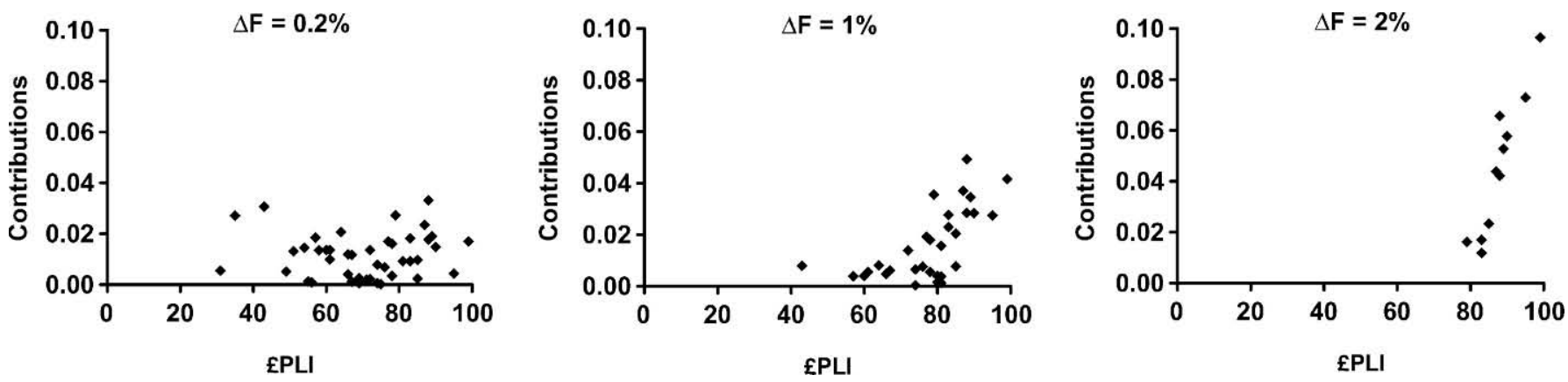

Figure 2. Association between optimized contributions and £PLI index scores for 3 levels of constraint on inbreeding $(\Delta \mathrm{F})$ for selected males for Top 2000 . 
average level of inbreeding is somewhat uninformative, as it is dependent on the definition of the base year. Since the UK population started many decades before the base year of 1940 used in this study, average inbreeding coefficients are likely to be underestimated. Another reason for the underestimation is that grade up animals with incomplete pedigrees are also included. Animals with fewer than 3 complete generations of pedigree information account for only about $4 \%$ of the animals born in 2002, but it is possible that some bias comes from this source, as they may be inbred but assumed noninbred.

On the other hand, the rate of inbreeding provides a more meaningful description of inbreeding in the population. Over the last decade or so, inbreeding has increased at a rate of $0.17 \% / y r$. This equates to an increase of about $1 \%$ per generation. Slightly higher rates of inbreeding have been observed in the United States (AIPL, 2003) and Canadian (CDN, 2003) Holstein populations over the same time period. The increase in the rate of inbreeding in the early 1990s could be due to the collective nature of a number of factors occurring in UK dairy cattle around this time. A few very popular foreign-related sires started to exert a large influence on the breed, which quickly led to an increase in the relatedness of the population. Also around this time, the individual animal model was implemented as the genetic evaluation method of choice for dairy cattle in the United Kingdom. The individual animal model utilizes information from all relatives, thereby increasing the coselection of related animals, especially within "good" families. As the large importation of North American Holstein sires in the United Kingdom continues, it is likely that the trend in inbreeding will follow that of the United States and Canada, but with a lag of about one generation unless measures are taken to control inbreeding.

For dairy cattle breeders, one of the consequences of inbreeding will be losses due to inbreeding depression. Inbreeding depression has been described in production traits (e.g., Smith et al., 1998; Thompson et al., 2000;), but it is expected to be greater in nonproduction traits such as fertility and disease resistance. The inclusion of health and fertility traits in national selection indices indicates the increasing importance of these traits in the breeding goal, and it is necessary to examine inbreeding depression in these traits. Wall et al. (2003) found that inbreeding had a significant effect on traits such as calving interval, days to first service, nonreturn rate at $\mathrm{d} 56$, and the number of inseminations required for a cow to become pregnant in the UK dairy population. More significantly, they found that the effect of inbreeding was more severe at higher levels of inbreeding. This study has shown that nearly $96 \%$ of the cow population is now inbred to some degree, and a greater frequency of cows is present in higher inbreeding classes than in 1990 (Table 1). If the current trend continues, it is likely that a greater proportion of animals will be in the higher inbreeding classes, leading to greater inbreeding depression for these traits.

Significant inbreeding depression suggests that the current rate of inbreeding should be controlled or reduced if further losses are to be avoided. In recent years, optimization tools have been developed to restrict the rate of inbreeding in a population. Furthermore, optimized selection has resulted in higher genetic gains at the same rates of inbreeding, or in lower rates of inbreeding at the same gain when compared to conventional truncation selection (Villanueva et al., 2004; Avendaño et al., 2003). Weigel and Lin (2002) examined the use of optimized selection in 5 dairy breeds in the United States. Unsurprisingly, they found a reduction in genetic merit as the constraint on inbreeding became more severe. This would be expected, as more animals with lower breeding values are required to achieve more severe constraints. However, they did not compare optimized selection with current selection to assess its ability to achieve higher genetic gains at the same level of inbreeding. The application of optimized selection will not only depend on the ability to achieve a predefined rate of inbreeding, but also to increase genetic gains compared to current selection strategies. The structure of dairy cattle breeding means that long-term inbreeding will depend mostly on the relationship between the sires and dams of young AI bulls (Weigel and Lin, 2002) rather than the use of particular bulls to breed cows. In this study, we applied optimized selection to groups of selection candidates whose offspring are potential young AI bulls. Expected average pedigree index scores when the contributions of the selected candidates were optimized were compared to the expected pedigree index scores using observed contributions at the same rate of inbreeding. For the top 2000 males, the expected $\Delta \mathrm{F}$ from observed parental contributions was $2 \%$, and the average $£$ PLI was $£ 62$. At the same $\Delta \mathrm{F}$ when only male contributions were optimized, an average £PLI of $£ 72$ was achieved. The increased gain is the result of increasing the selection intensity on the sires using optimized selection. At the same $\Delta \mathrm{F}$, only 11 sires are chosen with optimized selection compared to 173 sires originally used. A similar benefit of optimized selection can be seen when the rates of inbreeding are compared at the same genetic gain. Optimized selection achieves the same average pedigree index score $(£ P L I=62)$ as the observed genetic gain, but $\Delta \mathrm{F}$ was only $0.2 \%$. This is 10 -fold less than the observed $\Delta \mathrm{F}$, demonstrating that current levels of gain can be achieved at lower rates of inbreeding. When contributions of both sexes 
were optimized, the expected average pedigree index of the offspring was $£ 102$. This type of optimization can sometimes lead to unrealistic numbers of males and females selected. However, in the situation in which only a few hundred male offspring are required, it may be possible to generate the required number of offspring with the numbers of males and females selected using optimized selection for both sexes. For example, to achieve a $\Delta \mathrm{F}$ of just $0.1 \%, 42$ males and 47 females would be required. The expected merit of their offspring would be $£ 90$ - a $45 \%$ increase in genetic gain compared with the observed gain. With AI and the use of advanced reproductive techniques, such as in vitro fertilization and embryo sexing, it would be possible to generate enough offspring to achieve these goals.

Optimized selection was successful in meeting the constraint on $\Delta \mathrm{F}$ for all levels. In agreement with Weigel and Lin (2002), the number of animals selected increased and genetic gain decreased the more severe the constraint on $\Delta \mathrm{F}$. To meet the more stringent constraints, the relationship between the selection candidates needs to be reduced. This means selecting more animals (i.e., allocating more nonzero contributions to animals with lower average index scores) and equalizing contributions of selected animals (see Figure 2).

The application of optimized selection requires the determination of an acceptable rate of accumulation of inbreeding. In a finite population, such as the Holstein, inbreeding is inevitable, but the optimum rate of inbreeding is unknown. The optimum rate of inbreeding might be determined by balancing genetic progress and inbreeding depression. For example, Weigel and Lin (2002) calculated an optimum level of inbreeding of $7 \%$ in the next generation for lifetime net merit adjusted for inbreeding. Genetic merit decreased for levels of inbreeding greater than 7\%, as inbreeding depression had a greater impact at these increased levels. For levels less than $7 \%$, more animals are required to meet the constraint, and genetic gain is reduced.

Results from applying optimized selection could differ, depending on the index used to select candidates. In this study, we chose to look at the 2 indices currently used for the UK dairy industry. Whereas a high correlation exists between the 2 indices, the number of selected animals required to meet the constraints varied, especially at more severe constraints on $\Delta \mathrm{F}$. For example, at $\Delta \mathrm{F}=2 \%, 11$ males were selected for both indices. However, 6 extra males were required to meet a constraint of $0.2 \%$ for £PLI. Inclusion of more traits, such as fertility, will likely lead to a greater reranking of sires among the different indices and, hence, a change in the optimal contributions of selection candidates to meet a specific constraint.
In the United States, the average relationship of a particular sire to the female population is available. This can be used by breeders to choose outcross sires if they wish to control inbreeding on an individual herd basis. However, this will likely have little impact on the overall rate of inbreeding in the population as a whole. There is also the danger that if outcross sires of high merit become available, they may be heavily used in the future. As concluded by both Avendaño et al. (2003) and Weigel and Lin (2002), the application of optimized selection requires a coordinated policy on the use of selected candidates and also on breeding objectives. This may require the cooperation of pedigree breeders and AI organizations to ensure that the correct animals and their contributions are identified, which may prove to be challenging. However, the benefits of using optimized selection not only for controlling the rate of inbreeding but also to achieve higher genetic gains are clear.

\section{CONCLUSIONS}

Whereas inbreeding levels in the UK dairy population are not at alarming levels yet, it appears likely that the current rise in inbreeding will continue. The increase in inbreeding will lead to greater inbreeding depression and possibly increased prevalence of recessive genetic disorders in the population. Routine estimation of rates of inbreeding and inbreeding depression, especially for nonproduction traits, should be conducted to monitor the effects of inbreeding. The use of optimized selection provides a useful tool to manage the rate of accumulation of inbreeding in the population. More importantly, however, is that use of optimized selection can increase genetic gains for a given rate of inbreeding or attain similar genetic gains at much lower rates of inbreeding when compared to current selection strategies.

\section{ACKNOWLEDGMENTS}

The authors would like to thank Holstein UK for contributing data to the study. The authors are grateful to Sue Brotherstone for helpful discussions and comments. This work was funded by the Scottish Executive Environment \& Rural Affairs Department (SEERAD). J. Kearney would also like to acknowledge the National University of Ireland for studentship support.

\section{REFERENCES}

Animal Improvement Programs Laboratory (AIPL). 2003. Subject: Inbreeding. http://www.aipl.arsusda.gov/. Accessed Dec. 1, 2003. Avendaño, S. A., B. Villanueva, and J. A. Woolliams. 2003. Expected increases in genetic merit from using optimized contributions in 
two livestock populations of beef cattle and sheep. J. Anim. Sci. 81:2964-2975.

Canadian Dairy Network (CDN). 2003. Subject: Inbreeding. http:// www.cdn.ca. Accessed Dec. 1, 2003.

Cassell, B. G., V. Adamec, and R. E. Pearson. 2003. Effect of incomplete pedigrees on estimates of inbreeding and inbreeding depression for days to first service and summit milk yield in Holsteins and Jerseys. J. Dairy Sci. 86: 2967-2976.

Goddard, M. E., and C. Smith. 1990. Optimum number of bull sires in dairy cattle breeding. J. Dairy Sci. 73:1113-1122.

Grundy, B., B. Villanueva, and J. A. Woolliams. 1998. Dynamic selection procedures for constrained inbreeding and their consequences for pedigree development. Genet. Res. Camb. 72:159168.

MacCleur, J. W., A. J. Boyce, B. Dyke, L. R. Weitkamp, D. W. Pfennig, and C. J. Parsons. 1983. Inbreeding and pedigree structure in Standardbred horses. J. Hered. 74:394-399.

Milk Development Council Evaluations (MDC). 2003. Subject: Technical bulletin. http://www.mdcevaluations.co.uk. Accessed Dec. $1,2003$.

Meuwissen, T. H. E. 1997. Maximizing the response of selection with a predefined rate of inbreeding. J. Anim. Sci. 75:934-940.

Meuwissen, T. H. E., and Z. Luo. 1992. Computing inbreeding coefficients in large populations. Genet. Sel. Evol. 24:305-313.

Roughsedge, T., S. Brotherstone, and P. M. Visscher. 1999. Quantifying genetic contributions to a dairy cattle population using pedigree analysis. Livest. Prod. Sci. 60:359-369.
Smith, L. A., B. G. Cassell, and R. E. Pearson. 1998. The effects of inbreeding on the lifetime performance of dairy cattle. J. Dairy Sci. 81:2729-2737.

Swalve, H. H., F. Rosner, and W. Wemheuer. 2003. Inbreeding in the German Holstein cow population. Proc. 54th Annual Meeting of Eur. Assoc. Anim. Prod., Rome, Italy 9:17.

Thompson, J. R., R. W. Everett, and N. L. Hammerschmidt. 2000. Effects of inbreeding on production and survival in Holsteins. J. Dairy Sci. 83:1856-1863.

Villanueva, B., R. Pong-Wong, J. A. Woolliams, and S. Avendaño. 2004. Managing genetic resources in selected and conserved populations. In Farm Animal Genetic Resources. G. Simm, B. Villanueva, K. D. Sinclair, and S. Townsend, ed. BSAS Occasional Publication No. 30. Nottingham Univ. Press, Nottingham, UK.

Wall, E., S. Brotherstone, J. F. Kearney, J. A. Woolliams, and M. P. Coffey. 2003. Effect of including inbreeding, heterosis and recombination loss in prediction of breeding values for fertility traits. Interbull Bull. 31:117-121.

Weigel, K. A. 2001. Controlling inbreeding in modern breeding programs. J. Dairy Sci. 84(E. Suppl.):E177-E184.

Weigel, K. A., and S. W. Lin. 2002. Controlling inbreeding by constraining the average relationship between parents of young bulls entering AI progeny test programs. J. Dairy Sci. 85:2376-2383.

Young, C. W., and A. J. Seykora. 1996. Estimates of inbreeding and relationship among registered Holstein females in the United States. J. Dairy Sci. 79:502-505. 